\title{
DIFFERENTIAL INDUCTION OF PROINFLAMMATORY CYTOKINES IN PRIMARY MOUSE ASTROCYTES AND MICROGLIA BY CORONAVIRUS INFECTION
}

\author{
Dongdong Yu and Xuming Zhang*
}

\section{INTRODUCTION}

Mouse hepatitis virus (MHV) can infect rodents and cause digestive and central nervous system (CNS) diseases. The severity of the diseases is influenced by both viral and host factors. The strain JHM is highly neurovirulent while A59 is low neurovirulent, although both strains cause encephalitis and demyelination. By contrast, strain MHV-2 is non-neurovirulent, causing only mild meningitis but no encephalitis and demyelination. ${ }^{1}$ Studies have shown that the viral spike protein is the major determinant for neurovirulence. Recombinant A59/JHM-S, which contains the JHM spike gene in the A59 genomic background, exhibited a neurovirulent phenotype in C57BL/6 mice similar to that of $\mathrm{JHM}^{2}$ while A59/MHV2-S (Penn-98-1), which contains the spike of MHV-2, caused acute encephalitis but did not cause demyelination. ${ }^{3}$ Thus, the spike can modulate the viral pathogenic phenotype. The host immune system also plays a critical role in the onset and progression of the CNS disease. There is clear evidence showing that MHVinduced CNS diseases are often accompanied by lymphocyte infiltration in the CNS. Some studies using immunodeficient mice showed that the demyelination induced by JHM is largely immune-mediated, whereas others using RAG1-/- mice found that the immune system is not absolutely required for the demyelination when mice are infected with A59. ${ }^{4,5}$ Therefore, the precise role of individual components of the immune system in the demyelination process is not known.

The role of proinflammatory cytokines in the pathogenesis of CNS diseases has been studied both in vitro and in vivo. Indeed, significant upregulation of proinflammatory cytokines and chemokines has been observed during the course of CNS disease of mice infected with JHM and A59. Infection of primary mouse astrocytes and microglia with A59 significantly induced proinflammatory cytokines, especially TNF- $\alpha$ and IL- 6 , while infection with MHV-2 did not. ${ }^{6}$ Thus, the ability of the virus strains to induce cytokines

*University of Arkansas for Medical Sciences, Little Rock, Arkansas 72205. 
correlates with their neuropathogenic phenotypes, suggesting a role for proinflammatory cytokines in the disease process. However, removing TNF- $\alpha$ from mice through depletion with antibodies did not block or reduce demyelination induced by $\mathrm{JHM}^{7}$ thus negating the role for TNF- $\alpha$ in the demyelination process.

To further clarify this controversial issue and to determine whether the neurovirulent determinant spike protein is responsible for the induction of the cytokines in glial cells, we used Penn-98-1 in comparison with A59 and MHV-2 to determine proinflammatory cytokines in primary astrocytes and microglia. We found that a significant level of TNF$\alpha$ and IL- 6 was induced by A59 but not by MHV-2. Unexpectedly, Penn-98-1 induced TNF- $\alpha$ and IL-6 at a much higher level than that by A59. Our results thus suggest that the spike is not responsible for the induction of the proinflammatory cytokines in glial cells and that these proinflammatory cytokines might be associated with acute encephalitis but not with demyelination seen in A59-infected animals.

\section{MATERIALS AND METHODS}

\subsection{Viruses, Cells, and Reagents}

The following viruses were used: MHV-A59 and MHV-2 (kindly provided by Michael Lai, USC), and the recombinant virus Penn-98-1 (kindly provided by Ehud Lavi, University of Pennsylvania). Penn-98-1 contains the genome of A59 with the replacement of S gene by MHV-2. ${ }^{3}$ All viruses were propagated in DBT cells, and were purified by ultracentrifugation through a $30 \%(\mathrm{wt} / \mathrm{vol})$ sucrose cushion at $27,000 \mathrm{rpm}$ for $3 \mathrm{~h}$ at $4^{\circ} \mathrm{C}$ (Beckman). The virus pellets were resuspended in phosphate-buffered saline (PBS). For preparation of mouse primary astrocytes and microglia, neonatal C57BL/6 mice were sacrificed. Astrocytes and microglia were isolated from the mouse brains by using a technique exploiting the differential adherence characteristics of astrocytes and microglia. ${ }^{8}$ The purity of both astrocyte and microglia cell preparations was verified by immunofluorescence staining with antibodies specific to glial fibrillary acidic protein (GFAP) (Dako cytomation) and CD11b (Biosource), respectively. This isolation procedure routinely yielded cell populations with a purity of $>95 \%$. The MAPK p38 inhibitor SB203580 and the JNK inhibitor SP600125 were purchased from Biosource and were dissolved in DMSO.

\subsection{Cytometric Beads Array (CBA) Assay and Western Blot Analysis}

To quantify the cytokines produced and secreted into the culture medium, $50 \mu \mathrm{lof}$ virus-infected or mock-infected culture medium were subjected to CBA assay according to the manufacturer's instruction (BD Sciences). The mouse inflammatory cytokine beads array kit includes 6 proinflammatory cytokines (TNF- $\alpha$, IL-6, IL-10, IL-12p70, MCP-1, IFN- $\gamma$ ). To determine JNK activation, Western blot was carried out according to the manufacturer's instruction (Cell Signaling Technology Inc., CA). 


\section{RESULTS AND DISCUSSION}

\subsection{Cytokine Response to MHV Infection in Primary Astrocytes and Microglia}

We determined the cytokine proteins in primary mouse astrocytes and microglia following infection with A59, MHV-2, and Penn-98-1 for $24 \mathrm{~h}$, and compared them with those of mock-infected cells. We found that MCP-1 was significantly induced to a similar level by all 3 viruses, whereas no induction of IL-12p70, IFN- $\gamma$, and IL-10 was detected in cells infected with the 3 viruses. TNF- $\alpha$ and IL-6 were significantly higher in A59infected than in MHV-2-infected cells. However, to our surprise, both TNF- $\alpha$ and IL-6 were much higher in Penn-98-1-infected cells than in A59-infected cells (Fig. 1).

Since the only difference between A59 and Penn-98-1 is the spike protein, the result suggests that the spike is not responsible for the induction of TNF- $\alpha$ and IL-6. Moreover, since Penn-98-1 does not cause demyelination in mice, ${ }^{3}$ our finding suggests the proinflammatory cytokines such as TNF- $\alpha$ and IL-6 might not be sufficient for causing demyelination.

\subsection{Involvement of MAPK Pathways in the Regulation of Cytokine Induction in Primary Astrocytes by MHV Infection}

In general, cytokines are regulated via MAPK pathways. To determine whether and which specific MAPK pathway(s) is involved in regulation of TNF- $\alpha$ and IL-6 induction by MHV infection, we used the p38 inhibitor SB203580 $(40 \mu \mathrm{M})$ and JNK inhibitor SP600125 $(20 \mu \mathrm{M})$ to treat astrocytes $1 \mathrm{~h}$ prior to infection, and determined the cytokines in the medium by CBA. The result showed that the p38 inhibitor significantly inhibited the induction of IL- 6 but not of TNF- $\alpha$, while the JNK inhibitor blocked the induction of both IL- 6 and TNF- $\alpha$. This result suggests that the p38 MAPK pathway is involved in regulation of IL-6 induction whereas the JNK MAPK pathway is involved in both IL-6 and TNF- $\alpha$ induction by MHV infection. To further determine the biological relevance of JNK activation to MHV pathogenesis, we compared the activation of JNK phosphorylation by the 3 MHV strains with different pathogenic phenotypes. Astrocytes were infected with A59, MHV2, Penn-98-1 at 5 m.o.i. or mock infected. Cells were collected at $24 \mathrm{~h}$ p.i., and Western blot analysis was carried out to detect JNK phosphorylation. Results showed that A59 and Penn-98-1, but not MHV2, induced JNK phosphorylation (data not shown). Because A59 and Penn-98-1 can induce TNF- $\alpha$ and IL-6 and cause acute encephalitis in mice whereas MHV-2 cannot, our findings suggest that the induction of the proinflammatory cytokines by A59 and Penn-98-1 may contribute to the acute encephalitis by the 2 viruses.

\subsection{Viral Replication Is Required for the Induction of TNF- $\alpha$ and for the Activation of the JNK Signaling Pathway}

To determine whether MHV replication was required for the induction of TNF- $\alpha$ and IL-6, we used UV light-irradiated or live viruses to infect primary astrocytes. At $24 \mathrm{~h}$ p.i., TNF- $\alpha$ and IL- 6 in the culture medium were determined by CBA. We found that there was no induction of TNF- $\alpha$ in cells infected with the UV-irradiated virus. In contrast, UV-irradiated virus still induced IL- 6 to a level similar to that induced by the live virus 
A.

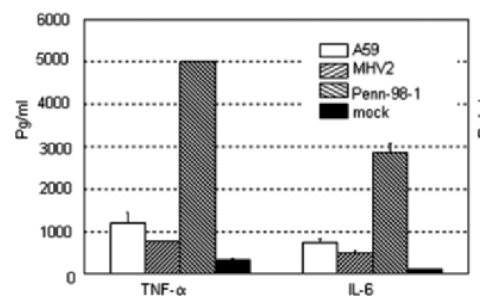

B.

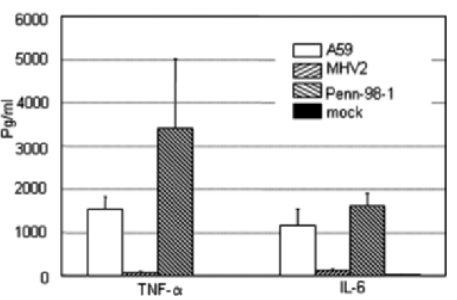

Figure 1. TNF- $\alpha$ and IL-6 production in primary astrocytes (A) and microglia (B) by MHV infection. Data indicate the means of the results from three independent experiments in the same pool of cells and the standard deviation of the means, which are representative of at least 3 pools of cells ( 9 independent experiments).

(data not shown). Taken together, these results demonstrate that MHV replication is required for TNF- $\alpha$ induction. We further determined if virus replication is required for the JNK pathway activation. c-Jun is the down-stream transcription factor of JNK that phosphorylates c-Jun. Our results showed that c-Jun was phosporylated by infection with A59 and Penn-98-1 but not by infection with MHV-2, and that c-Jun phosphorylation required viral replication (data not shown). These results thus correlate the activation of the JNK pathway with the induction of TNF- $\alpha$ by MHV infection.

\section{ACKNOWLEDGMENTS}

This work was supported by grants from the National Institutes of Health (AI 47188 and NS47499).

\section{REFERENCES}

1. Das Sarma, J., Fu, L., Hingley, S. T., Lai, M. M. C., and Lavi, E., 2001, Sequence analysis of the S gene of recombinant MHV-2/A59 coronaviruses reveals three candidate mutations associated with demyelination and hepatitis, J. Neurovirol. 7:432-436.

2. Navas, S., and Weiss, S. R., 2003, Murine coronavirus-induced hepatitis: JHM genetic background eliminates A59 spike-determined hepatotropism, J. Virol. 77:4972-4978.

3. Das Sarma, J., Fu, L., Tsai, J. C., Weiss, S. R., and Lavi, E., 2000, Demyelination determinants map to the spike glycoprotein gene of coronavirus mouse hepatitis virus, J. Virol. 74:9206-9213.

4. Houtman, J. J., and Fleming, J. O., 1996, Dissociation of demyelination and viral clearance in congenitally immunodeficient mice infected with murine coronavirus JHM, J. Neurovirol. 2:101-110.

5. Matthews, A. E., Lavi, E., Weiss, S. R., and Paterson, Y., 2002, Neither B cells nor T cells are required for CNS demyelination in mice persistently infected with MHV-A59, J. Neurovirol. 8:257-264.

6. Li, Y., Fu, L., Gonzales, D. M., and Lavi, E., 2004, Coronavirus neurovirulence correlates with the ability of the virus to induce proinflammatory cytokine signals from astrocytes and microglia, J. Virol. 78:33983406.

7. Stohlman, S. A., Hinton, D. R., Cua, D., Dimacali, E., Sensintaffar, J., Hofman, F. M., Tahara, S. M., and Yao, Q., 1995, Tumor necrosis factor expression during mouse hepatitis virus-induced demyelinating encephalomyelitis, J. Virol. 69:5898-5903.

8. Esen, N., Tanga, F. Y., DeLeo, J. A., Kielian, T., 2004, Toll-like receptor 2 (TLR2) mediates astrocyte activation in response to the Gram-positive bacterium Staphylococcus aureus, J. Neurochem. 88:746-758. 